\title{
Proliferative, Migratory, and Transition Properties Reveal Metastate of Human Amnion Cells
}

\author{
Lauren Richardson*† and Ramkumar Menon*
}

From the Division of Maternal-Fetal Medicine and Perinatal Research, * Department of Obstetrics and Gynecology, and the Department of Neuroscience, Cell Biology and Anatomy, ${ }^{\dagger}$ The University of Texas Medical Branch at Galveston, Galveston, Texas

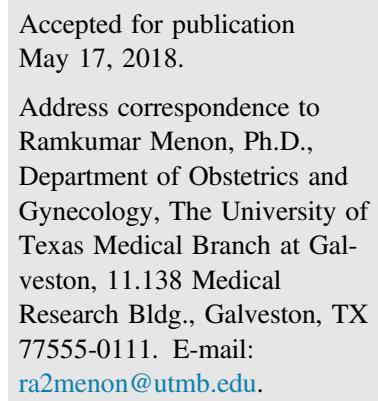

\begin{abstract}
Amnion epithelial cell (AEC) shedding causes microfractures in human placental membranes during gestation. However, microfractures are healed to maintain membrane integrity. To better understand the cellular mechanisms of healing and tissue remodeling, scratch assays were performed using primary AECs derived from normal term not in labor membranes. AECs were grown under different conditions: i) normal cultures (control), ii) oxidative stress (OS) induction by cigarette smoke extract (CSE), iii) co-treatment of CSE and antioxidant $\mathrm{N}$-acetyl-L-cysteine, and iv) treatment with amniotic fluid (AF). Cell migration time and distance, changes in intermediate filament (cytokeratin-18 and vimentin) expressions, and cellular senescence were determined. Control AECs in culture exhibited a metastate with the expression of both cytokeratin-18 and vimentin. During healing, AECs proliferated, migrated, and transitioned from epithelial to mesenchymal phenotype with increased vimentin. Wound healing was associated with mesenchymal to epithelial transition (MET). CSE-induced OS and senescence prevented wound healing in which cells sustained mesenchymal state. $\mathrm{N}$-acetyl-L-cysteine reversed CSE's effect to aid wound closure through MET. AF accelerated cellular transitions and healing. Our data suggest that AECs undergo epithelial to mesenchymal transition during proliferation and migration and MET at the injury site to promote healing. AF accelerated whereas OS diminished cellular transitions and healing. OS-inducing pregnancy risk factors may diminish remodeling capacity contributing to membrane dysfunction, leading to preterm birth. (Am J Pathol 2018, 188: 2004-2015; https://doi.org/10.1016/ j.ajpath.2018.05.019)
\end{abstract}

During pregnancy, placental membranes (fetal or amniochorionic membranes) provide mechanical support and immune tolerance for the growing fetus until their disruption at term. ${ }^{1}$ Placental membranes are composed of amnion epithelial cells (AECs) and chorion trophoblast cells, connected by a collagen-rich extracellular matrix (ECM) that also contains amnion mesenchymal stromal cells. $^{2-4}$ Membranes expand with the developing embryo, undergoing continuous remodeling throughout gestation to maintain structural integrity and accommodate fetal growth. $^{4-6}$ Membranes, specifically the amnion layer, are highly elastic, allowing them to withstand the stretch and strain endured during fetal growth. ${ }^{7}$ We recently reported that the membranes undergo telomere-dependent ${ }^{8}$ senescence via oxidative stress (OS)-induced p38 mitogen-activated protein kinase signaling. ${ }^{9,10}$ Both human and animal models suggest that development of senescence and senescence-associated inflammation are progressive events that peak at term as the membranes reach their longevity. ${ }^{10-13}$ Senescent membranes fail to remodel, ${ }^{14}$ lose their structural integrity, and generate various inflammatory signals that can cause transition of a quiescent uterus to an active (in labor) status. Thus, membranes can alternatively support pregnancy or promote labor. Because premature senescence is associated with preterm delivery, ${ }^{15,16}$ understanding the mechanisms that regulate

Supported by NIH National Institute of Child Health and Human Development grant 1R01HD084532-01A1 (R.M.). L.R. is an appointed predoctoral trainee in the Environmental Toxicology Training Program supported by NIH National Institute of Environmental Health Sciences grant T32ES007254 and administered through The University of Texas Medical Branch in Galveston, Texas.

Disclosures: None declared. 
placental membrane homeostasis will help to elucidate causes of preterm birth (PTB) and preterm prelabor rupture of membranes (pPROM), two major pregnancy complications.

Placental membrane cells are pluripotent, with stem-like characteristics (self-renewal and capable of differentiation when stimulated under appropriate conditions) and the ability to remodel through a well-balanced inflammatory process during gestation. This is supported by self-healing of wounds after fetoscopy, ${ }^{17,18}$ as well as minimal incidence of placental membrane rupture after invasive procedures, such as amniocentesis, where incision injuries are often selflimiting, repaired, and heal spontaneously. ${ }^{19}$ A recent report by Carvalho et $\mathrm{al}^{20}$ noted reparative activity at the placental membrane surgical sites after open fetal surgery. This activity was characterized by a significant increase in nascent collagen synthesis, tissue remodeling, and repair of suture site, mechanisms likely to prevent amniotic fluid (AF) leakage and to maintain pregnancy. Human amniotic membranes are shown to promote healing of iatrogenic placental membrane defect sites. ${ }^{21-23}$ However, ambiguity ${ }^{24}$ and lack of understanding of the mechanisms involved in wound healing has hindered the advancement of intrauterine interventions to prevent various fetal anomalies. Effective interventions for pPROM, a disease of the placental membranes that affects approximately $40 \%$ of all spontaneous PTB that results from failure to heal membranes, are also elusive because of gaps in knowledge of the mechanisms of membrane remolding.

Recently, we reported certain unique features in placental membranes from term labor (TL) delivery compared with term not in labor (TNIL) delivery. Multiphoton microscopy of membranes showed amnion cell shedding and/or puckering, basement membrane and ECM degradation, and migration of cells through tunnels in the ECM. ${ }^{14}$ These structures are collectively called microfractures, and TL microfractures are distinct from those in TNIL. Microfractures are more frequent in TL than TNIL membranes from cesarean sections. In vitro, TNIL membranes exposed to OS inducers, increased microfractures, and their morphometry were similar to those seen in TL. ${ }^{14}$ Similarly, microfractures were more frequent in pPROM and PTB with intact membranes than gestational age-matched membranes from preeclampsia. ${ }^{25} \mathrm{We}$ postulate that microfractures are areas of membrane remodeling and are increased in response to OS at term or preterm. At term, this could be considered as a normal physiologic response, ensuring fetal and membrane/ placental delivery. However, failure to repair and heal microfractures may predispose them to rupture and/or delivery in PTB, when pregnancy-associated risk factors increase intra-amniotic OS.

Although the mechanisms of wound healings are not well characterized, several studies have found wound healing properties of placental membranes, specifically the amnion layer. In a recent study, Janzen et $\mathrm{al}^{26}$ proposed transition of
AECs to mesenchymal cells [epithelial to mesenchymal transition (EMT)], a process associated with inflammation and accelerated by inflammatory mediators. In support of this study, Mogami et al ${ }^{27}$ reported that small ruptures of the placental membrane (generated by a 26-gauge needle) closed within 72 hours in a preclinical mouse model on day 15 of gestation, whereas limited healing capacity was associated with large ruptures (generated by a 20 -gauge needle). Small ruptures induced transient up-regulation of cytokines, whereas large ruptures elicited sustained upregulation of proinflammatory cytokines in the placental membranes. Fetal macrophages facilitated localized inflammation, EMT, and epithelial cell migration in this model, enabling wound healing.

On the basis of these reports, it is hypothesized that OS inducers compromise membrane microfracture remodeling and healing capacity, leading to persistence of wounds or fractures that cause dysfunctional membranes with inflammation. To further investigate the kinetics and mechanisms of the wound healing and transition capacity of AECs under normal and OS conditions, an in vitro scratch assay was performed and their transition during the wound healing process studied. In addition, it was demonstrated how OS compromises and AF nurtures the healing capacity of amnion cells. We report that AECs from term membranes can proliferate, undergo EMT for cell propagation, and heal a scratch when cells regain epithelial phenotype through mesenchymal to epithelial transition (MET).

\section{Materials and Methods}

\section{Study Design}

This study protocol was approved by the institutional review board at The University of Texas Medical Branch at Galveston, TX, as an exempt protocol for using discarded placenta after normal term cesarean deliveries (project 69693). No subject recruitment or consent was required for this study.

\section{AEC Culture}

Primary AECs were isolated from TNIL amnion (approximately $10 \mathrm{~g}$ ), peeled from the chorion layer, and dispersed by successive treatments with $0.125 \%$ collagenase and $1.2 \%$ trypsin. All cell culture reagents were purchased from Sigma-Aldrich (St. Louis, MO), and details of AEC isolation protocols can be found in previous reports. ${ }^{8,9,28}$ This isolation method has been optimized to eliminate amnion mesenchymal cell contamination as verified by cytologic evaluation of all the preparations on seeding. The dispersed cells were plated in a 1:1 mixture of Ham's F12/Dulbecco's modified Eagle's medium, supplemented with $10 \%$ heatinactivated fetal bovine serum, $10 \mathrm{ng} / \mathrm{mL}$ of epidermal growth factor, $2 \mathrm{mmol} / \mathrm{L}$ L-glutamine, $100 \mathrm{U} / \mathrm{mL}$ of 
penicillin $\mathrm{G}$, and $100 \mathrm{mg} / \mathrm{mL}$ of streptomycin at a density of 3 million to 5 million cells per T75 and incubated at $37^{\circ} \mathrm{C}$ with $5 \% \mathrm{CO}_{2}$ until $80 \%$ to $90 \%$ confluence was achieved. Although there are limitations to culturing AECs with epidermal growth factor and fetal bovine serum, as primary cells they require such growth factors to survive in vitro. Growth factors in AEC media did not promote additional proliferation and migration of cells as documents by the OS treatments.

\section{AF Collection}

TNIL AF was used from our existing biobanks. AF samples were collected from women undergoing cesarean delivery by transabdominal amniocentesis. Samples were immediately centrifuged three times at $3000 \times g$ to remove all cells and particulate debris. Aliquots of supernatant were stored at $-80^{\circ} \mathrm{C}$ until further use. Inclusion criteria included elective cesareans after an uncomplicated pregnancy before initiation of labor. Exclusion criteria included history of antimicrobial treatments during pregnancy, any surgical procedures, smoking during pregnancy, positive screening for group B Streptococcus between 35 and 37 weeks of gestation, body mass index $>25$, prior history of preterm labor or other complications of pregnancy, and bacterial vaginosis.

\section{Scratch Assay and Cell Culture Treatments}

Passage 1 (P1) AECs were seeded at approximately $80 \%$ confluence in four-well coverslips and incubated at $37^{\circ} \mathrm{C}$ with $5 \% \mathrm{CO}_{2}$ for 24 hours. AECs were then serum starved for 1 hour, rinsed with sterile $1 \times$ phosphate-buffered saline (PBS), and then scratched evenly down the middle of the well, in a straight line, with a $200-\mu \mathrm{L}$ pipet tip. Cells were washed with sterile $1 \times$ PBS four times to remove any cell debris. ${ }^{29}$ To measure cellular proliferation versus migration, P1 AECs were plated for scratch assay after being inubated with $5 \mu \mathrm{mol} / \mathrm{L}$ of carboxyfluorescein succinimidyl ester for 20 minutes, rinsed with PBS, and resuspended. Carboxyfluorescein succinimidyl ester stains cells green and only loses its intensity after cell division. This allowed identifying cells that proliferated (lost their initial green fluorescent protein intensity) versus migrated (maintined their initial intensity) to seal the wound.

\section{Exposure of Scratch to OS Conditions and Normal Term $\mathrm{AF}$}

To test the effect of OS on wound healing and the effect of $\mathrm{AF}$ in nurturing AECs, scratch wounds were treated with one of the following: i) control Dulbecco's modified Eagle's medium/F12 media, ii) OS inducer cigarette smoke extract (CSE) 1:25 media, ${ }^{9}$ iii) CSE and antioxidant $N$-acetyl-Lcysteine (NAC; $15 \mathrm{mmol} / \mathrm{L}$; Sigma-Aldrich), or iv) AF samples from TNIL cesareans (TNIL AF; 1:1 dilution in culture media) and incubated at $37^{\circ} \mathrm{C}, 5 \% \mathrm{CO}_{2}$, and $95 \%$ air humidity for up to 38 hours.

\section{Immunocytochemical Localization of Intermediate Filaments CK-18 and Vimentin}

AEC immunocytochemical staining for vimentin $(3.7 \mu \mathrm{L} / \mathrm{mL}$; ab92547; Abcam, Cambridge, MA) and cytokeratin-18 (CK-18) $(1 \mu \mathrm{L} / \mathrm{mL}$; ab668; Abcam) were performed at 1 hour, 10 hours, 22 hours, and 38 hours after the initial scratch was made. Manufacturer's instructions were used to calculate staining dilutions to ensure uniform staining. After each time point, cells were fixed with $4 \%$ paraformaldehyde, permeabilized with $0.5 \%$ Triton $\mathrm{X}$, and blocked with $3 \%$ bovine serum albumin in PBS before incubation with primary antibodies overnight at $4^{\circ} \mathrm{C} .{ }^{28}$ This protocol is adequate to remove nonspecific binding of primary antibodies in our system. After washing with PBS, slides were incubated with Alexa Fluor 488- and Alexa Fluor 594-conjugated secondary antibodies (Life Technologies, Carlsbad, CA) and diluted 1:1000 in PBS for 1 hour in the dark. Slides were washed with PBS, treated with NucBlue Live ReadyProbes Reagent (Life Technologies), and then mounted using Mowiol 4 to 88 mounting medium (Sigma-Aldrich).

\section{Microscopy and Image Analysis}

Images of scratch sites were recorded and media were collected 1 hour, 22 hours, and 38 hours after the initial scratch. Bright-field microscopy images were captured using a Nikon Eclipse TS100 microscope $(10 \times)$ (Nikon, Melville, NY). Five regions of interest per condition were used to determine the overall cell morphologic features. Confocal microscopy images were captured using a Zeiss 880 confocal microscope $(10 \times)$ (Zeiss, Oberkochen, Germany). Five random regions of interest per field were used to determine red (CK-18) and green (vimentin) fluorescence intensity. Uniform laser settings, brightness, contrast, and collection settings were matched for all images collected. Images were not modified (brightness, contrast, and smoothing) for intensity analysis. ImageJ software version $1.51 \mathrm{~J}$ (NIH, Bethesda, MD; http://imagej.nih.gov/ij) was used to measure vimentin and CK-18 staining intensity from two focal planes of three different regions per treatment condition at each time point. Image analysis was conducted in triplicate for all cell experiments.

\section{SA- $\beta$-Gal Assay}

The presence of the senescence-associated $\beta$-galactosidase (SA- $\beta$-Gal) distinguishes senescent cells from quiescent cells. $^{9,30-32}$ This enzymatic activity is distinct from the ubiquitous acidic $\beta$-galactosidase and can be detected at $\mathrm{pH}$ 6.0 using the chromogenic substrate X-Gal. Senescent cells were identified using a histochemical staining kit (Sigma-Aldrich), with blue cells visualized by light 
microscopy 38 hours after treatment. Four regions of interest per condition were captured by bright-field microscopy using a Nikon Eclipse TS100 microscope $(10 \times)$. Uniform brightness, contrast, and collection settings were matched for all images collected. Image modifications (brightness, contrast, and smoothing) were applied to entire image sets using Lightroom software version 7.4 (Adobe, San Jose, CA) and ImageJ software. The total number of cells in each bright-field was counted, and the number of SA- $\beta$-Gal-positive cells was divided over the total amount of cells to show the percentage of SA- $\beta-\mathrm{Gal}-$ stained cells per condition.

\section{Total Collagen Type 1 Assay}

A total collagen type 1 assay kit was purchased through Abcam, and kit instructions were modified to run cell culture media. Cell supernatants were mixed with equal volumes of $\mathrm{NaOH}(10 \mathrm{~N})$ and hydrolyzed individually at $120^{\circ} \mathrm{C}$ for 1 hour. Samples were then cooled on ice, and the hydrolysate was neutralized by adding an equivalent volume of $\mathrm{HCl}(10 \mathrm{~N})$. Supernatants were decolorized by adding $4 \mathrm{mg}$ of activated charcoal to the neutralized hydrolysate and centrifuged at $10,000 \times g$ for 5 minutes to remove precipitate and activated charcoal. Clear supernatant $(10 \mu \mathrm{L})$ was added to a $96-$ well plate. The standards and samples were evaporated by heating at $65^{\circ} \mathrm{C}$ on a hot plate. Oxidation mix $(100 \mu \mathrm{L})$ was added and the plate incubated at room temperature for 20 minutes. Developer $(50 \mu \mathrm{L})$ was then added to each well and kept at $37^{\circ} \mathrm{C}$ for 5 minutes. 4-(Dimethylamino)benzaldehyde concentrate solution $(50 \mu \mathrm{L})$ was added and the plate placed on a hot plate at $65^{\circ} \mathrm{C}$ for 45 minutes. Absorbance of each sample was then measured at OD $560 \mathrm{~nm}$ using a microplate reader. Standard curves were developed with samples of known quantities of recombinant proteins that were provided by the manufacturer. Sample concentrations were determined by relating the absorbance values obtained to the standard curve by linear regression analysis. Manufacturer's instructions were followed to calculate the hydrolysate and total collagen production in micrograms per microliter.

\section{Enzyme-Linked Immunosorbent Assay for Inflammatory Marker IL-8}

Enzyme-linked immunosorbent assay was performed for IL8 (Biosource International, Camarillo, CA, and Luminex Corporation, Austin, TX) as an indicator of general inflammation. As a chemokine, IL-8 has been associated with EMT, ${ }^{33}$ senescence, ${ }^{31}$ and wound healing. ${ }^{34}$ Standard curves were developed with duplicate samples of known quantities of recombinant proteins that were provided by the manufacturer. Sample concentrations were determined by relating the absorbance values that were obtained to the standard curve by linear regression analysis.

\section{Statistical Analysis}

Data were analyzed for significant differences using GraphPad Prism software version 7 (GraphPad Software, San Diego, CA). One-way analysis of variance followed by the Tukey multiple comparison posthoc test or the $t$-test were used for comparison of the studied groups. Statistically significant difference is indicated by a $P<0.05$.

\section{Results}

\section{Innate State of AECs}

To determine the innate transition state of primary AECs in culture, immunocytochemical analyses of epithelial and mesenchymal intermediate filaments, CK-18, and vimentin, respectively, were performed, followed by confocal microscopy. To rule out any deleterious effects of processing of placental membranes for cell cultures, AECs at passage (P) 0 and P1 were immunostained. P0 AECs stained 24 hours after isolation showed expression of both CK-18 and vimentin and had round morphologic features, which is as expected and normal before attachment to the culture plates. Cells attained the typical cobblestone morphologic features of epithelial cells in P1, with enhanced expression of both intermediate filaments (Figure 1). Freshly harvested AECs contained both CK-18 and vimentin, two key components of epithelial and mesenchymal cells, suggesting that they were at an inbetween state of transition termed metastate.

\section{Migratory and Transition Potential of AECs}

The migratory potential of metastate AECs was studied under normal in vitro conditions. Wounds inflicted by scratch on AECs healed naturally within 38 hours that included AEC proliferation, migration, and transition (Figure 2, A and B, and Supplemental Figure S1). Specifically, cell morphologic changes and changes in the expression pattern of CK-18 and vimentin were examined to determine changes associated with wound healing. Co-expression of CK-18 and vimentin were seen before scratch and up to 10 hours after the initial wound. After 22 hours, dominance of vimentin-positive cells with mesenchymal morphologic features at the leading edge were seen (Figure 2C). Mesenchymal cells are more migratory, ${ }^{35}$ and the CK-18/vimentin staining pattern suggests that EMT promoted cellular migration to heal the wound. Wounds were healed within 38 hours, and examination of these sites showed dominance of intermediate filament CK- $18^{+}$ cells with a morphologic finding that indicated MET (Figure 2C). Confocal microscopic analysis confirmed that perinuclear vimentin-stained cells with epithelial morphologic features were predominant before scratch, whereas proliferating cells at leading edges showed increased vimentin staining at the periphery of cells (Figure 3, left 

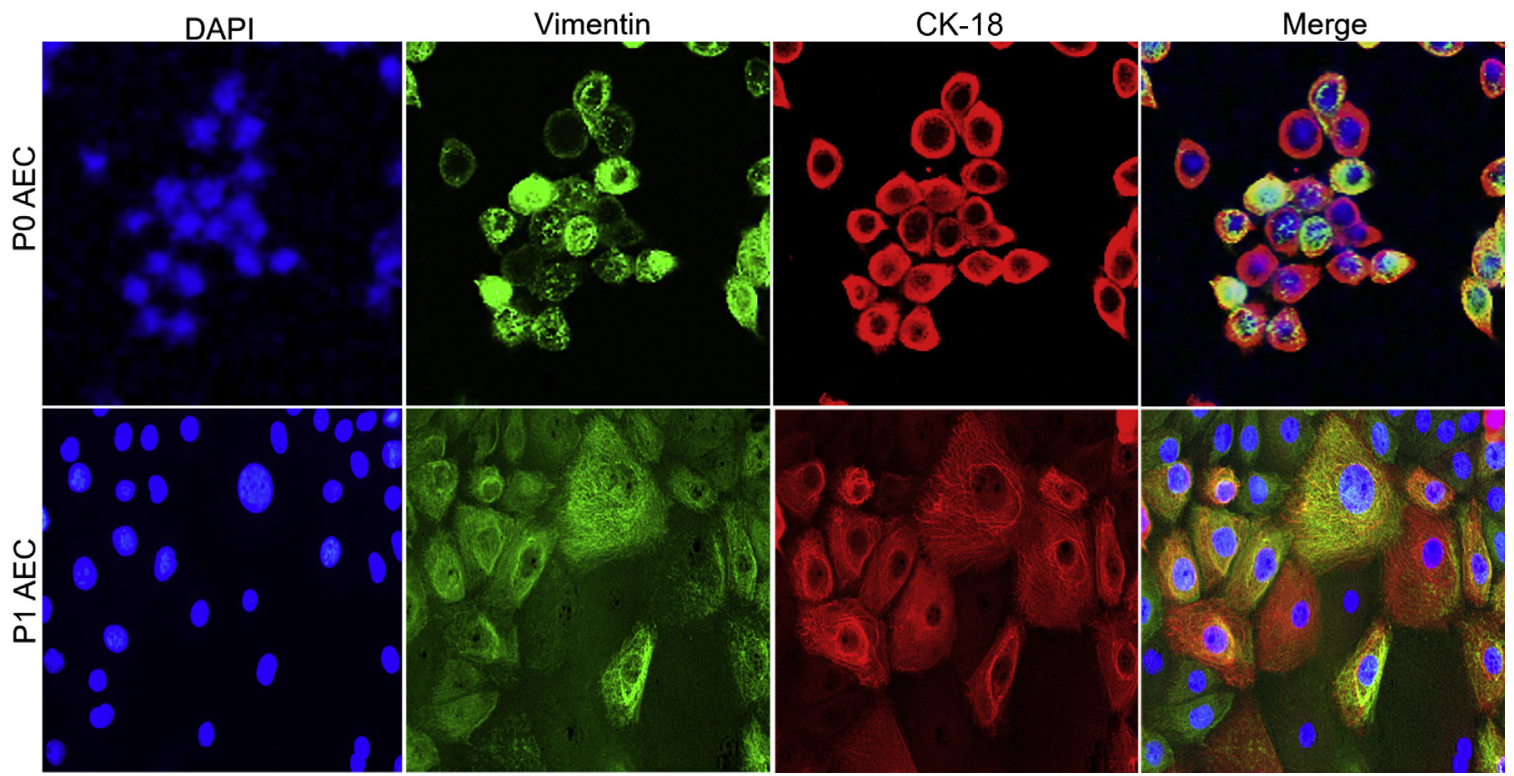

Figure 1 Characteristics of metastate amnion epithelial cells (AECs) in culture. Confocal microscopy showing vimentin (green) and cytokeratin-18 (CK-18) (red) localized in the cytoplasm of both passage 0 (PO) and passage 1 (P1) AECs. Perinuclear localization of vimentin and CK-18 is expected in P0 cells. Fully attached P1 cells show cytoplasmic localization of both vimentin and CK-18. Original magnification, $\times 63$.

column). After 38 hours, AECs with perinuclear vimentin and epithelial morphologic features dominated the healed wound. CK-18 was diffused thoroughout the cytoplasm and did not follow the same reorganization trend as vimentin during wound healing (Figure 3, right column). The EMT and MET not only produced differential expression of intermediate filaments but also caused cytoskeletal reorganization.

\section{OS Prevents Wound Closures}

Intra-amniotic OS and reactive oxygen radicals are required for cellular proliferation and fetoplacental growth. ${ }^{36}$ At term, increased OS and reduced antioxidant levels promote OS-induced cellular damage. ${ }^{1,9}$ To test the ability of AECs to undergo transition and their wound healing capacity under OS, we conducted scratch assays after exposing the

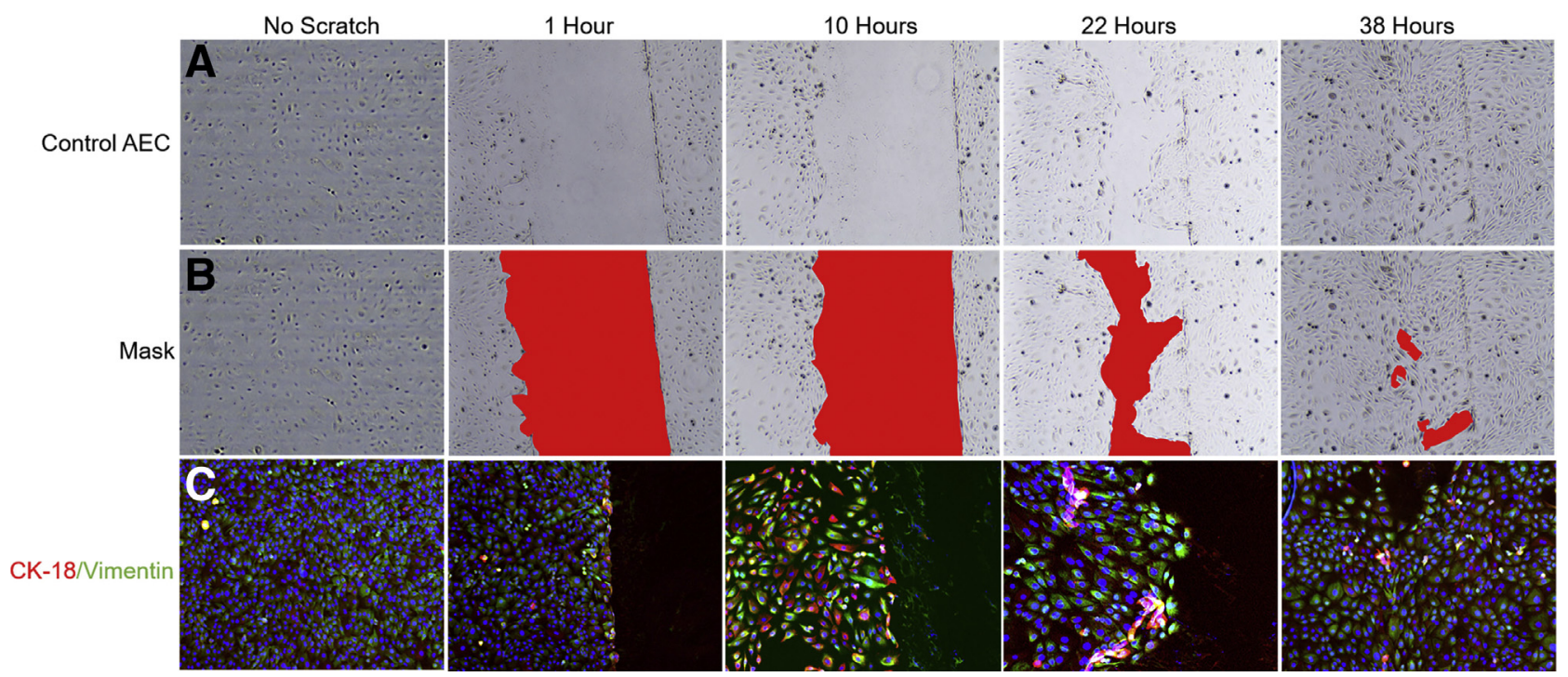

Figure 2 Scratch assay demonstrates that amnion epithelial cells (AECs) naturally heal wounds. A: Bright-field microscopy of AECs under normal cell culture conditions showing wound healing at 38 hours. B: A red mask was overlaid on the wound to highlight the area of AEC proliferation and migration throughout the 38-hour period. C: Fluorescence microscopy showing vimentin (green) and cytokeratin-18 (CK-18) (red) localized in the cytoplasm of AECs at various time points after scratch and in no-scratch control cells. A heterogeneous population of cells co-expressing both markers was recorded at 1 hour and 10 hours, as well as in the no-scratch control experiment. At 22 hours, AECs were predominantly vimentin positive at the leading edge. After healing of the wound at 38 hours, AECs reverted to metastate, co-expressing both intermediate filaments with dominance of CK-18. Original magnification, $\times 10$. 


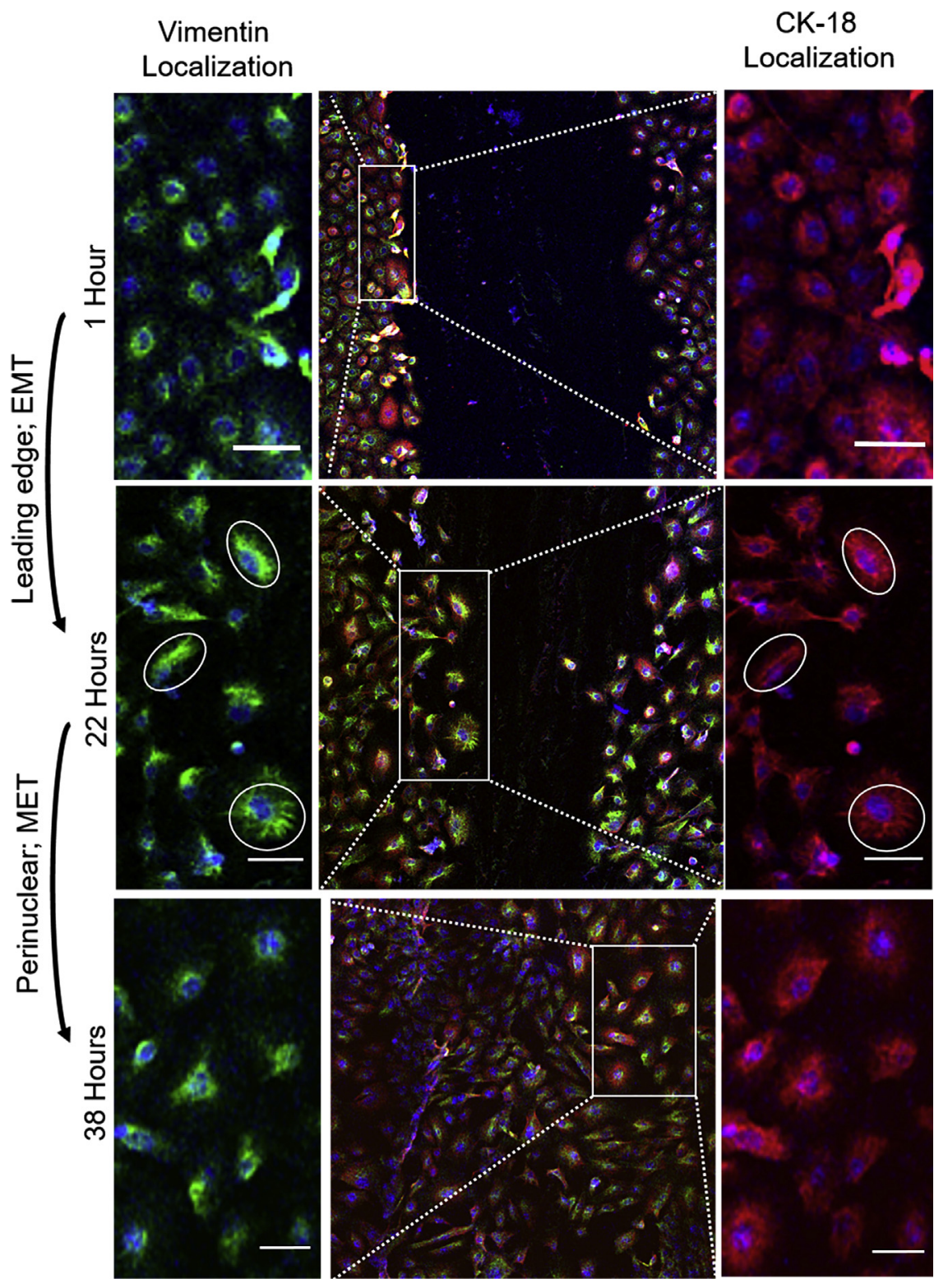

Figure 3 Vimentin relocalization during wound healing. Confocal microscopic images showing vimentin (green) and cytokeratin-18 (CK-18) (red) localized in amnion epithelial cell (AEC) healing a wound. At the scratch site (left column), vimentin is located in the perinuclear region of predominantly epithelial amnion cells. This localization shifts to the periphery of cells at the migratory edges, where cells start to acquire mesenchymal morphologic features, indicating epithelial to mesenchymal transition (EMT). White circles highlight vimentin localization on the leading edge. Wound healing site is characterized by perinuclear localization of vimentin along with cells acquiring epithelial morphologic features, suggesting mesenchymal to epithelial transition (MET). Right column: Although expression levels change, unlike vimentin, localization of CK-18 does not change during the EMT or MET process (white circles). Boxed areas in the center column are enlarged in both the left and right columns. Scale bars $=50 \mu \mathrm{mol} / \mathrm{L}$. Original magnification, $\times 10$. cells to CSE, an inducer of OS. ${ }^{9}$ The water-soluble portion of CSE is used exclusively as a laboratory reagent because it is a well-reported OS inducer and produces changes in AECs in culture to mimic fetal membranes at TL. Examination of various CSE components and their potential risk during pregnancy are beyond the scope of this study. CSE treatment of cells that mimicked OS experienced at TL and delivery prevented wound healing because the migrated distance between the two scratch edges was significantly shorter $(108.2 \pm 45.06 \mu \mathrm{m})$ in the CSE-treated group compared with controls $(867 \pm 96.01 \mu \mathrm{m})(P<0.0001)$ after 38 hours. OS caused by CSE was reversed by co-treatment with NAC. Co-treatment with NAC and CSE in culture media significantly improved wound healing because the distance traveled by cells to heal the wound was higher compared with CSE alone (655.4 $\pm 19.27 \mu \mathrm{m}$; $P<0.001$ ) (Figure 4, A and B).

\section{AF Accelerates Wound Healing}

The amniotic membrane, composed of a single layer of AECs, is constantly bathed in AF that contains but is not limited to growth factors, cytokines, and exosomes. ${ }^{37,38} \mathrm{We}$ tested the hypothesis that nutrient-rich AF can nourish and nurture amniotic membranes to heal any gaps generated by cell shedding. Scratch wounds were treated with TNIL AF because these samples have lower levels of reactive oxygen radicals and markers of OS compared with TL samples. ${ }^{1}$ Treatment with TNIL AF healed wound edges by inducing cell migration that covered a greater distance 

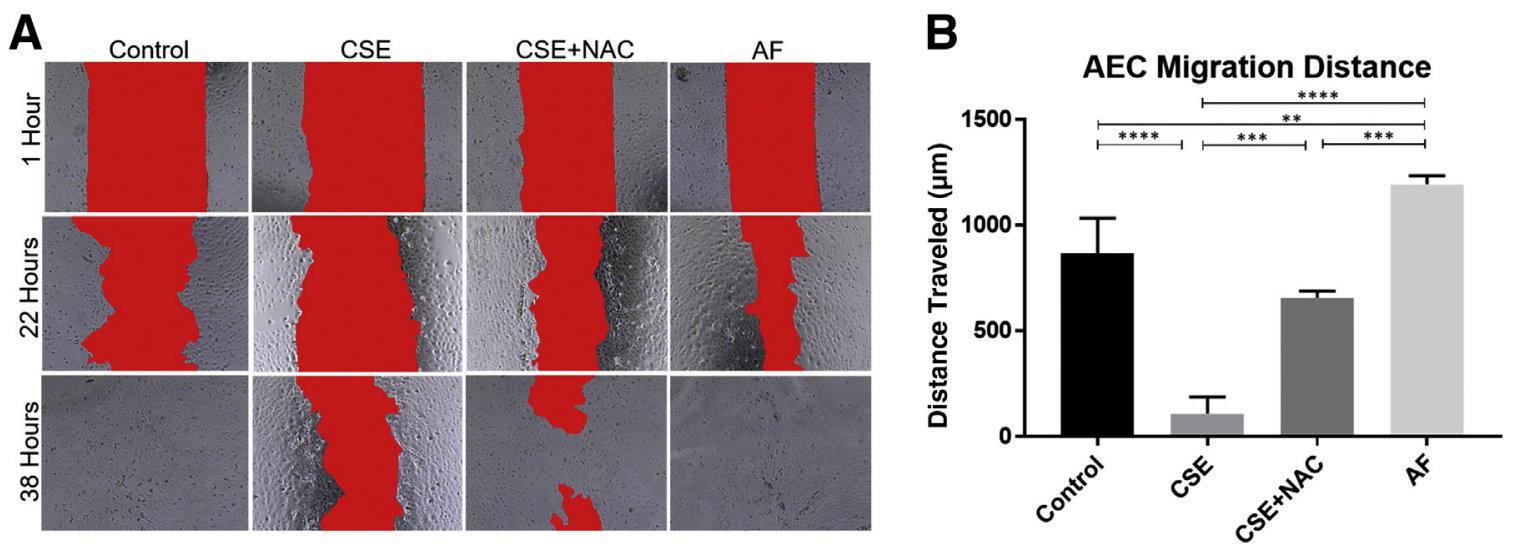

Figure 4 Documentation of cellular migration and wound healing under normal oxidative stress conditions and after treatment with amniotic fluid. A and B: A red mask was applied to help visualization of the wound field and distance was measured at different time points. Amnion epithelial cells (AECs) under normal cell culture conditions show their natural ability to heal a wound within 38 hours. Cigarette smoke extract (CSE) prevents wound healing, indicated by a migration distance between the two scratch edges that is significantly shorter $(108.2 \pm 45.06 \mu \mathrm{m})$ in CSE-treated group compared with controls $(867 \pm 96.01$ $\mu \mathrm{m})$ after 38 hours. Co-treatment with antioxidant $N$-acetyl-L-cysteine (NAC) significantly increases the distance traveled by cells to heal the wound $(655.4 \pm 19.27 \mu \mathrm{m})$ compared with CSE alone. Treatments with term not in labor amniotic fluid (AF) heals wound edges by increasing migration distance between the two scratch edges compared with controls $(1193 \pm 23.79 \mu \mathrm{m}$ versus $867 \pm 96.01 \mu \mathrm{m})$. Data are expressed as means \pm SD $(\mathbf{B})$. ${ }^{* *} P<0.01$, $* * * P<0.001$, and ${ }^{* * *} P<0.0001$. Original magnification, $\times 10(\mathbf{A})$.

between the two scratch edges compared with controls $(1193 \pm 23.79 \mu \mathrm{m}$ versus $867 \pm 96.01 \mu \mathrm{m} ; P<0.01)$, therefore supporting our hypothesis.

\section{OS Induces a Constant State of EMT}

Immunocytochemistry followed by confocal microscopy and ImageJ analysis of vimentin/CK-18 ratios was used to determine the transition potential of AECs during wound healing. On the basis of staining intensity, wound healing involved both EMT and reversal of this process (MET). Control cells ( $\mathrm{P} 1$ cells in normal culture conditions) healed the wound by undergoing EMT, visualized at 22 hours by high vimentin/CK-18 ratios at the leading edge $(P<0.0001)$, and MET, visualized at 38 hours by low vimentin/CK-18 ratios at healed edges $(P<0.0001)$ (Figure 5, A and B). CSE treatment did not heal the wound and induced a constant state of EMT (Figure 5C), which was demonstrated by high vimentin/CK-18 ratios at 22 and 38 hours (Figure 5, A and C). Co-treatment with NAC (EMT: $P<0.001$; MET: $P<0.01$ ) (Figure 5, A and D) improved wound healing similar to that of controls and AF (EMT: $P<0.01$; MET: $P<0.01$ ) (Figure 5 , A and E). These results support our hypothesis that OS can generate a static state in cells, preventing their transition to a normal state.

\section{OS Induces Senescence and Increases Collagen Production}

As mentioned in the opening paragraphs of this article, CSE causes senescence of AECs in culture after 24 hours, a phenomenon seen in membranes at TL and a condition associated with parturition. ${ }^{12,39}$ Therefore, whether CSE caused AEC senescence, preventing cell proliferation and migration, was tested. AECs were stained for SA- $\beta-G a l$ as a marker of senescent cells. Bright-field microscopy demonstrated a significantly higher amount of SA- $\beta$-Gal-positive cells (blue cells) after CSE treatment compared with controls $(P<0.05)$ (Figure 6, A and B). SA- $\beta$-Gal-positive cells were recorded at the wound site and throughout the general cell population of CSE-treated cells. Media samples were analyzed for total collagen production and inflammatory mediators to determine collagen concentration at the scratch site. CSE treatment significantly stimulated collagen production $(P<0.0001)$ compared with controls; cotreatment with NAC did not decrease collagen production to a statistically significant level (Figure 6C). AF treatment also significantly increased collagen concentration in the media compared with control AECs $(P<0.0001)$ (Figure 6D). Nascent collagen production can be considered as an inflammatory process associated with remodeling, and it is likely that OS-induced damage, as well as AF nurturing of cells to proliferate and migrate, is associated with collagen synthesis.

\section{Inflammation Is Associated with Wound Healing}

The proinflammatory cytokine IL- 8 was observed at the wound healing site. Although IL-8 was induced by CSE $(19.67 \pm 11.44 \mathrm{pg} / \mathrm{mL})$ after 38 hours, it was not significantly different from controls $(11.92 \pm 4.738 \mathrm{pg} / \mathrm{mL})$ (Figure 6E). Co-treatment of CSE with NAC $(12.13 \pm 5.947 \mathrm{pg} / \mathrm{mL})$ decreased IL-8, although this decrease was not significant (Figure 6E). Cellular senescence caused by CSE diminishes tissue remodeling capacity of AECs, even with a mild increase in local proinflammatory cytokines. IL-8 progressively increased in both 

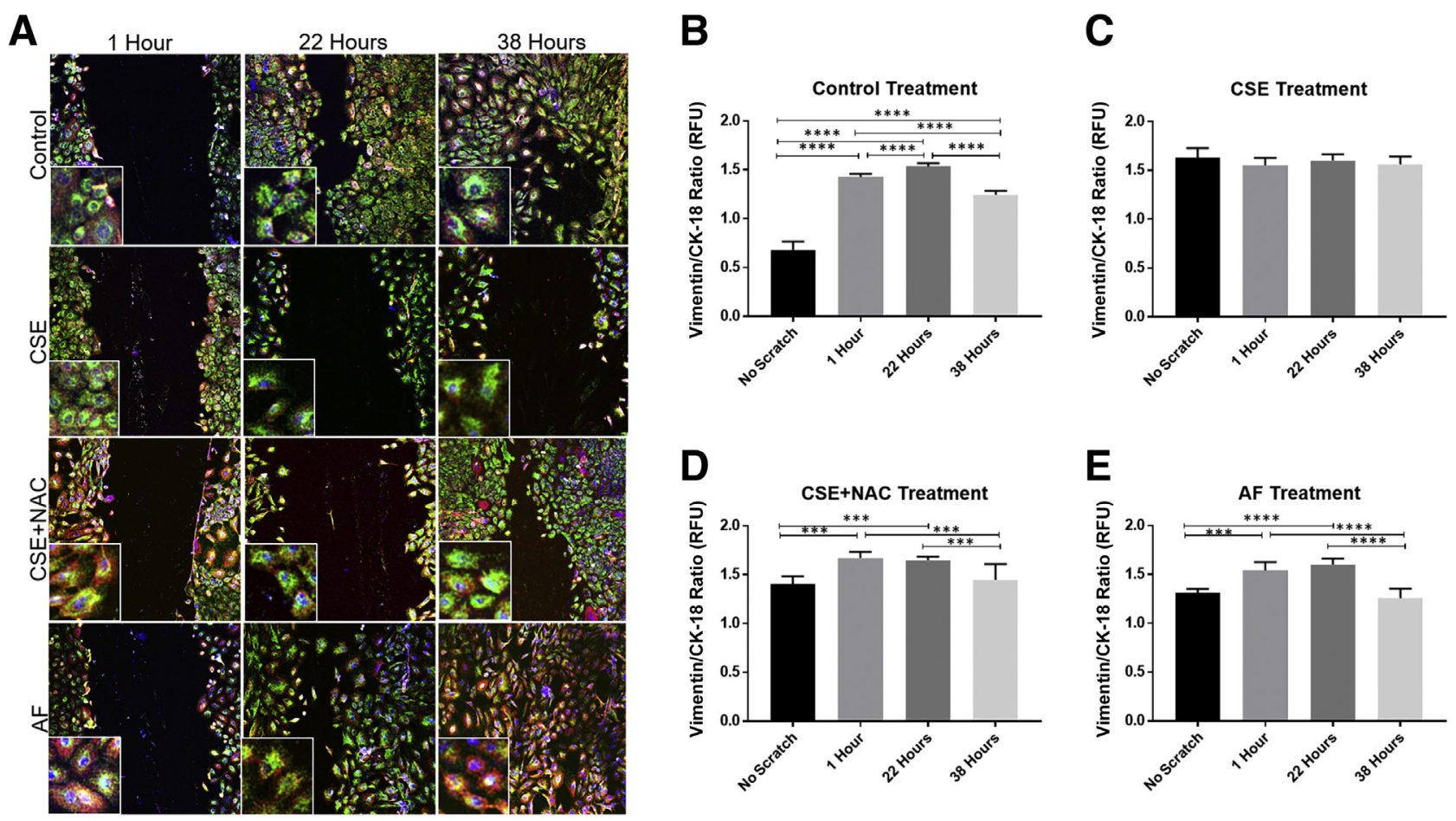

Figure 5 Amnion epithelial cells (AECs) heal wounds by undergoing epithelial to mesenchymal transition (EMT) and mesenchymal to epithelial transition (MET). A: Confocal microscopy image showing vimentin (green) and cytokeratin-18 (CK-18) (red) localization in AEC wound healing after different treatments. Insets show the overall staining pattern for each treatment. B: Under normal cell culture conditions, EMT was demonstrated at 22 hours with high vimentin/CK-18 ratios at the leading edge, and MET was demonstrated at 38 hours by low vimentin/CK-18 ratios at healed edges. C: Cigarette smoke extract (CSE) treatment induces a constant state of EMT compared with controls. D: The effect of CSE is inhibited by co-treatment with antioxidant $N$-acetyl-L-cysteine (NAC), which enables wound healing by promoting EMT, demonstrated at 22 hours by high vimentin/CK-18 ratios at the leading edge, and MET, demonstrated at 38 hours by low vimentin/CK-18 ratios at healed edges. E: Amniotic fluid (AF) treatments of cells produces changes similar to that of control. Wounds heal by EMT, demonstrated at 22 hours by high vimentin/CK-18 ratios at the leading edge, and MET, demonstrated at 38 hours by low vimentin/CK-18 ratios at healed edges. Data are expressed as means \pm SD (B). ${ }^{* *} P<0.001,{ }^{* * *} P<0.0001$. Original magnification, $\times 10$ (A). RFU, relative fluorescence unit(s).

control and AF-treated cell cultures, and a maximum value was seen at 38 hours. IL- 8 was significantly higher in AF-treated cells $(75.49 \pm 17.25 \mathrm{pg} / \mathrm{mL})$ at 38 hours compared with control cells $(17.25 \pm 8.45 \mathrm{pg} / \mathrm{mL})$ $(P<0.05)$ (Figure $6 \mathrm{~F})$, supporting the view that proinflammatory cytokines contribute to wound healing. The term AF samples that were used contained IL-8, which can confound the increased IL- 8 that occurs in the media. To control for this, IL-8 enzyme-linked immunosorbent assay of AF samples was conducted, and values were deducted from the media IL- 8 concentrations reported here. Wound healing is an inflammatory process, and IL- 8 chemoattracts neutrophils to aid wound healing. Accelerated healing of wounds by AF compared with controls is likely to be aided by increased localized proinflammatory cytokines, as indicated by IL- 8 increase in the media.

\section{Discussion}

OS and inflammation are physiologic components of pregnancy that help to promote tissue remodeling and membrane integrity maintenance. ${ }^{14}$ As previously reported by us, microfractures develop during membrane growth in gestation because of shedding of senescent or apoptotic cells, characterized by degradation of basement membranes and ECM collagen. ${ }^{14,25}$ However, these microfractures do not threaten pregnancy status or membrane integrity because they are healed by tissue and collagen remodeling. This process is aided by reactive oxygen radical-mediated signaling and localized inflammation similar to what is documented in wound healing. ${ }^{40}$ This physiologic process of tissue remodeling is likely to be aided by nutrient-rich AF and is a balanced process throughout pregnancy. However, at term, increased fetal metabolic demands and decreased fetomaternal antioxidants lead to OS build-up in the amniotic cavity, which perturbs remodeling. ${ }^{10}$ This build-up leads to a static state of EMT, senescence, and inflammation, preventing recycling of transitioned mesenchymal cells back to their epithelial state. In this study, these scenarios were tested by inflicting a wound in cultured AECs, and the ability to seal these sites of insult was investigated. Whether AECs are stem cells or cells that exhibit stemness within the right environment has been debated. Regardless, tissue regeneration properties are widely used in a variety of clinical fields to promote wound healing. ${ }^{18,41,42}$ In this 
A

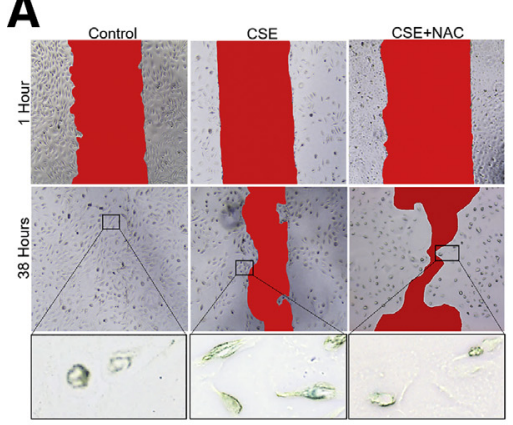

D

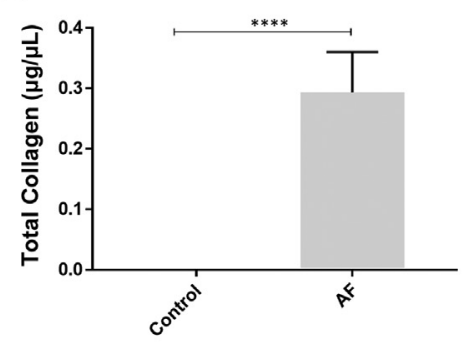

B

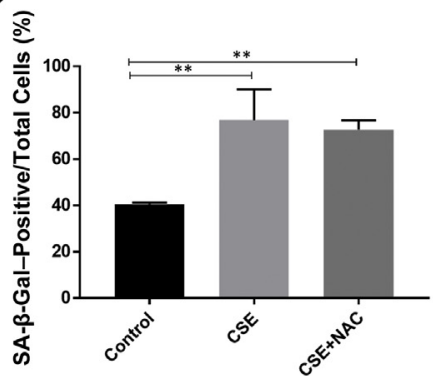

E

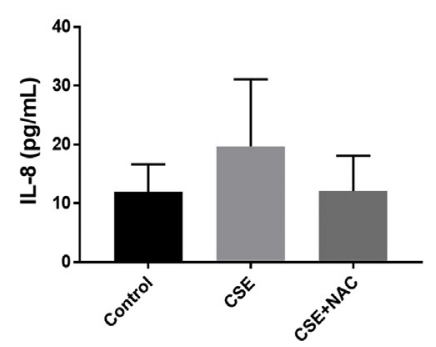

C

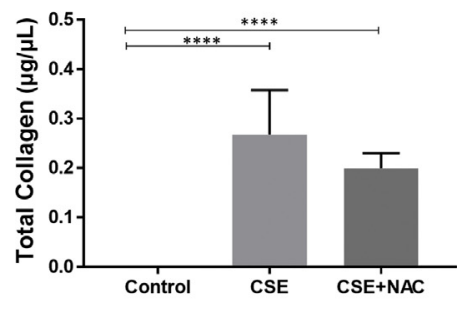

$\mathbf{F}$

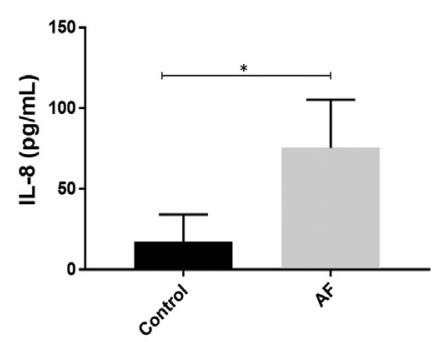

Figure 6 Epithelial to mesenchymal transition (EMT) is associated with senescence, collagen production, and inflammation. A and B: Senescenceassociated $\beta$-galactosidase (SA- $\beta$-Gal): cigarette smoke extract (CSE)-induced senescence (blue stain) in a larger population of amnion epithelial cells (AECs) healing the wound compared with controls, as seen in $\mathbf{A}$ and quantified in $\mathbf{B}$. Boxed areas in the middle row of $\mathbf{A}$ are shown at higher magnification in the bottom row. C and D: Collagen assay. C: CSE treatment significantly stimulates collagen production compared with controls, whereas antioxidant $\mathrm{N}$-acetyl-L-cysteine (NAC) decreases collagen production. D: Amniotic fluid (AF) treatment also significantly stimulates collagen production compared with controls. $\mathbf{E}$ and F: Chemokine IL-8 release. E: After 38 hours of exposure, CSE-treated AECs release more IL-8 than controls and CSE plus NAC. F: AF induces a significant amount of IL-8 production after healing the wound for 38 hours compared with controls. Data are expressed as means \pm SD (B-F). ${ }^{*} P<0.05$,

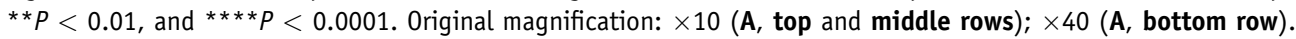

study, AECs were shown to proliferate, migrate, transition, and heal wounds in vitro (Supplemental Figure S1). In addition, the effect of excessive OS (similar to that seen at $\mathrm{TL})$ was tested in reducing migratory and proliferative (Supplemental Figure S1) capability of cells that could prevent wound healing and the capacity of AF to promote healing and wound closure during gestation. The key findings from this study are as follows: i) AECs at term gestation are not dead cells and are capable of proliferation, migration, transition, and self-renewal, which indicates their stemness; ii) amnion cells show innate expression of both epithelial and mesenchymal intermediate filaments, causing them to be in a metastate phenotype; iii) wound healing of amnion involves proliferation and migration of cells with cytoskeletal reorganization, while undergoing transition between EMT and MET; iv) AF expedites wound healing, aided by low levels of inflammation, stimulation of collagen synthesis, nourishment of AECs, and a likely reduction in OS; and v) on the contrary, OS induces a static state of EMT, senescence, and inflammation, which compromises wound healing. This effect of OS can lead to membrane dysfunction and mechanical instability. ${ }^{43}$ These changes can also predispose membranes to rupture, although this depends on the strength of redox imbalance. OS-induced outcome was partially reversed by co-treatment with NAC, thus supporting our hypothesis. In summary, we determined that AECs through a regulated EMT and MET transition (Figure 7) can heal gaps or microfractures, and this process is likely to be facilitated by AF. Increased OS at term $^{44}$ or in response to OS-inducing risk factors at preterm

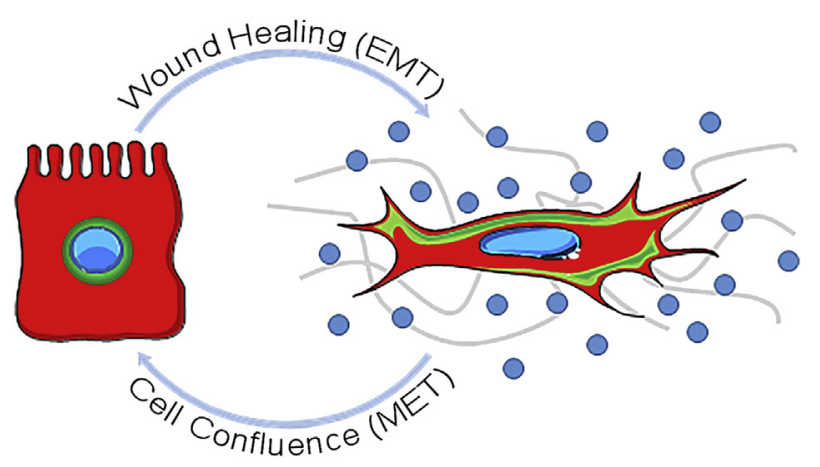

Figure 7 Amnion epithelial cells (AECS) undergo epithelial to mesenchymal transition (EMT) and mesenchymal to epithelial transition (MET) to remodel placental membranes. Diagram representing metastate AECs. AECS undergo EMT to heal a wound in which perinuclear vimentin (green) is increased and relocalized to periphery and leading edges of cells along with increased collagen (gray lines) and inflammatory mediator (blue circles) production. Transition is complete when epithelioid morphologic features are transformed to a mesenchymal shape. Wound closure is facilitated when cells revert back to MET, when epithelial morphologic features are reinstated to perinuclear localization of vimentin. This process promotes recycling and remodeling of $A E C s$ in the placental membranes. 
reduces the capacity for microfracture healing, which can predispose membranes to rupture.

AECs are unique because of their pluripotent status as stem cells, ${ }^{45}$ as well as their co-expression and localization of epithelial and mesenchymal markers. The ability of cells to express both epithelial and mesenchymal phenotypes was first reported by Savagner as a metastable phenotype, documented in his studies of Rac distribution in cancer cells. ${ }^{46,47}$ Here we describe AECs in an in-between state of epithelial and mesenchymal transition that we term a metastate, which is critical for AEC survival and maintenance of membrane integrity. Metastate is a response to changes in the membrane microenvironment, which helps maintain homeostasis and promotes wound healing during pregnancy. As previously reported, ${ }^{14}$ microfractures should not persist because they present likely channels for trafficking of shed AECs, immune cells, and leakage of AF. Although these characteristics have been overlooked in recent years, heterogenous populations of AECs expressing both vimentin and cytokeratin were first documented in the early 1980 s. ${ }^{48,49}$ Our ongoing studies support these findings, and additional EMT/METassociated changes, such as differential expression of Ecadherin and $\mathrm{N}$-cadherin expression, changes in stem cell transcription factors Nanog and OCT4, and other EMT/ MET transcription factors in cultured AECs and tissue explants, have been observed (L.R. and R.M., unpublished data). These metastate qualities of AECs are consistent with the expression of stem cells undergoing EMT, which is likely the reason that AECs are used as progenitor cells to generate various organs. ${ }^{50}$ These properties of AECs and amnion membranes are also used extensively for wound healing in burn patients. ${ }^{18,41,42,51}$

Similar to some cancer cells, AECs can undergo EMT to migrate followed by MET at a secondary site to perform specific functional roles, such as sealing the site to complete the repair process. These morphologic changes are also supported by the occurrence of vimentin localization (Figure 3) that has been documented to be vital for cell proliferation and migration in cancer cells. ${ }^{52,53}$ Cells with perinuclear vimentin staining with epithelial morphologic features were predominant before scratch and at healed edges, whereas leading and migratory edges had vimentin staining at the periphery of cells with mesenchymal morphologic features. This type of vimentin relocalization has been previously reported, and we were able to recapitulate this in our model. ${ }^{53,54} \mathrm{CK}-18$ localization was diffused throughout the cytoplasm and did not follow the same reorganization trend as vimentin during wound healing. This finding suggests that vimentin expression and localization play a vital role in transitioning AECs and can be used as a marker to document cells undergoing EMT or MET. The vimentin/CK-18 ratio may be used to monitor the state (epithelial versus mesenchymal) of cells before conducting experiments with AECs. These cells are always in a state of transition under specific environmental conditions, and their functional roles may be dependent on the dominant state of cells.

Throughout gestation, amnion membranes maintain their structural integrity by growing, healing microfractures, and adapting to changes in the endocrine and immune milieu. This adaptation is feasible because of their metastate qualities. The inflammatory environment observed in response to various treatments in our study indicates how AECs will respond to changes in the microenvironment. Here we document the existence of generalized inflammation at wound healing sites, consistent with the role of inflammation (ie, walling of the spread of an infection at the injury site and remodeling of tissue). Homogeneity in the inflammatory profile (collagen and chemokine increase irrespective of AF or CSE treatment) does not necessarily indicate functional harmony. In fact, these changes are indicative of distinct mechanisms in which the same inflammatory mediator may show uniformity in its concentration. Control and $\mathrm{AF}$ treatments tend to produce inflammation that is likely to promote remodeling ${ }^{33}$ and wound healing, ${ }^{34}$ whereas inflammation associated with CSE treatment is likely caused by cellular senescence, ${ }^{9}$ the latter being a detrimental factor for membrane survival. However, there are limitations in using some inflammatory mediators as biomarkers associated with pregnancy complications. On the basis of the underlying physiologic or pathophysiologic mechanisms, these biomarkers may be indicative of distinct underlying functions, and an increase in a cytokine/chemokine does not always reflect a pathologic condition.

Here we also document the natural ability of AECs to proliferate, migrate, and heal wounds via EMT and MET transition (Figure 2) and how AF supports the metastate qualities of AECs in enhancing wound closure (Figure 5). The data above suggest that nutrient-rich AF stimulates low levels of IL-8 (Figure 6), causing metastate AECs to undergo EMT and MET transition and promoting collagen synthesis to heal wounds. The levels of IL-8 seen during wound closure (approximately $60 \mathrm{pg} / \mathrm{mL}$ of IL-8) (Figure 6) are much lower than IL-8 levels reported in TNIL (2941 pg/mL) and $\mathrm{TL}(5571 \mathrm{pg} / \mathrm{mL}) \mathrm{AF}^{31}$ suggesting that treatments with AF can stimulate low levels of IL-8 that do not harm the cells but help them to remodel. This study did not examine specific factors involved in AF promotion of cellular transitions and/ or its remodeling properties, although $\mathrm{AF}$ has various growth factors, cytokines, and exosomes with a potential role in wound healing. ${ }^{37,38}$ Analysis of the specific AF constituent responsible for promoting AECs properties described here is beyond the scope of this work. We postulate that this wellbalanced process of cellular transition, collagen production, and inflammation plays a role in the healing of placental membranes after fetal surgery in women who have a balanced intrauterine redox status. However, exposure of placental membranes to excessive OS, at term or preterm, induces a static state of EMT, causing senescence and senescenceassociated inflammation that will prevent repair capabilities. 
The CSE data support this concept that OS could be a contributor of membrane rupture at term or preterm gestation, specifically in cases of pPROM, where an increased number of microfractures ${ }^{14}$ or lack of microfracture healing can compromise fetal membrane integrity. Although lack of remodeling, senescence, and inflammation are likely to be physiologic requirements for promoting parturition at term, all these factors could also cause premature rupture of the membranes leading to PTB.

In summary, we report certain unique features of fetal AECs. These cells exist in a metastate, undergoing constant transition that will help to maintain membrane structural and mechanical integrity. Stemness of AECs and their remodeling capabilities are aided by nourishment by AF. Excessive OS disrupts transitions and the remodeling process and can contribute to membrane dysfunction. We have already shown pathways of senescence and how senescence can disrupt membrane integrity. Herein, we provide additional information on the process by which membrane healing can be disrupted by senescent cells. Understanding the mechanisms and mediators of EMT and MET will increase our knowledge of the contribution of membranes to human pregnancy and parturition.

\section{Acknowledgments}

L.R. designed and performed experiments, analyzed data, and wrote the manuscript; R.M. conceived the project, designed experiments, provided funding, helped with data analysis and interpretation, and prepared the manuscript.

\section{Supplemental Data}

Supplemental material for this article can be found at https://doi.org/10.1016/j.ajpath.2018.05.019.

\section{References}

1. Menon R: Human fetal membranes at term: dead tissue or signalers of parturition? Placenta 2016, 44:1-5

2. Richardson L, Vargas G, Brown T, Ochoa L, Trivedi J, Kacerovský M, Lappas M, Menon R: Redefining 3dimensional placental membrane microarchitecture using multiphoton microscopy and optical clearing. Placenta 2017, 53:66-75

3. Jabareen M, Mallik AS, Bilic G, Zisch AH, Mazza E: Relation between mechanical properties and microstructure of human fetal membranes: an attempt towards a quantitative analysis. Eur J Obstet Gynecol Reprod Biol 2009, 144 Suppl 1:S134-S141

4. Mauri A, Ehret AE, Perrini M, Maake C, Ochsenbein-Kolble N, Ehrbar M, Oyen ML, Mazza E: Deformation mechanisms of human amnion: quantitative studies based on second harmonic generation microscopy. J Biomech 2015, 48:1606-1613

5. Menon R, Bonney EA, Condon J, Mesiano S, Taylor RN: Novel concepts on pregnancy clocks and alarms: redundancy and synergy in human parturition. Hum Reprod Update 2016, 22:535-560

6. Perrini M, Mauri A, Ehret AE, Ochsenbein-Kolble N, Zimmermann R, Ehrbar M, Mazza E: Mechanical and microstructural investigation of the cyclic behavior of human amnion. J Biomech Eng 2015, 137:061010

7. Gupta A, Kedige SD, Jain K: Amnion and chorion membranes: potential stem cell reservoir with wide applications in periodontics. Int J Biomater 2015, 2015:274082

8. Polettini J, Behnia F, Taylor BD, Saade GR, Taylor RN, Menon R: Telomere fragment induced amnion cell senescence: a contributor to parturition? PLoS One 2015, 10:e0137188

9. Menon R, Boldogh I, Urrabaz-Garza R, Polettini J, Syed TA, Saade GR, Papaconstantinou J, Taylor RN: Senescence of primary amniotic cells via oxidative DNA damage. PLoS One 2013, 8: e83416

10. Bredeson S, Papaconstantinou J, Deford JH, Kechichian T, Syed TA, Saade GR, Menon R: HMGB1 promotes a p38MAPK associated non-infectious inflammatory response pathway in human fetal membranes. PLoS One 2014, 9:e113799

11. Hadley EE, Richardson LS, Torloni MR, Menon R: Gestational tissue inflammatory biomarkers at term labor: a systematic review of literature. Am J Reprod Immunol 2017, 79

12. Bonney EM, Bonney EA, Krebs K, Saade G, Kechichian T, Trivedi J, Huaizhi Y, Menon R: Differential senescence in feto-maternal tissues during mouse pregnancy. Placenta 2017, 43:26-34

13. Sheller-Miller S, Lei J, Saade G, Salomon C, Irina B, Menon R: Fetomaternal trafficking of exosomes in murine pregnancy models. Front Pharmacol 2016, 7:432

14. Richardson LS, Vargas G, Brown T, Ochoa L, Sheller-Miller S, Saade GR, Taylor RN, Menon R: Discovery and characterization of human amniochorionic membrane microfractures. Am J Pathol 2017, $187: 2821-2830$

15. Menon R: Oxidative stress damage as a detrimental factor in preterm birth pathology. Front Immunol 2014, 5:567

16. Menon R, Boldogh I, Hawkins HK, Woodson M, Polettini J, Syed TA, Fortunato SJ, Saade GR, Papaconstantinou J, Taylor RN: Histological evidence of oxidative stress and premature senescence in preterm premature rupture of the human fetal membranes recapitulated in vitro. Am J Pathol 2014, 184:1740-1751

17. Gratacos E, Sanin-Blair J, Lewi L, Toran N, Verbist G, Cabero L, Deprest J: A histological study of fetoscopic membrane defects to document membrane healing. Placenta 2006, 27:452-456

18. Papadopulos NA, Klotz S, Raith A, Foehn M, Schillinger U, Henke J, Kovacs L, Horch RE, Biemer E: Amnion cells engineering: a new perspective in fetal membrane healing after intrauterine surgery? Fetal Diagn Ther 2006, 21:494-500

19. Borgida AF, Mills AA, Feldman DM, Rodis JF, Egan JF: Outcome of pregnancies complicated by ruptured membranes after genetic amniocentesis. Am J Obstet Gynecol 2000, 183:937-939

20. Carvalho NS, Moron AF, Menon R, Cavalheiro S, Barbosa MM, Milani HJ, Ishigai MM: Histological evidence of reparative activity in chorioamniotic membrane following open fetal surgery for myelomeningocele. Exp Ther Med 2017, 14: $3732-3736$

21. Papanna R, Mann LK, Tseng SC, Stewart RJ, Kaur SS, Swindle MM, Kyriakides TR, Tatevian N, Moise KJ Jr: Cryopreserved human amniotic membrane and a bioinspired underwater adhesive to seal and promote healing of iatrogenic fetal membrane defect sites. Placenta 2015, 36:888-894

22. Quintero RA, Morales WJ, Allen M, Bornick PW, Arroyo J, LeParc G: Treatment of iatrogenic previable premature rupture of membranes with intra-amniotic injection of platelets and cryoprecipitate (amniopatch): preliminary experience. Am J Obstet Gynecol 1999, 181:744-749

23. Mann LK, Papanna R, Moise KJ Jr, Byrd RH, Popek EJ, Kaur S, Tseng SC, Stewart RJ: Fetal membrane patch and biomimetic adhesive coacervates as a sealant for fetoscopic defects. Acta Biomater 2012, 8:2160-2165

24. Papanna R, Mann LK, Moise KJ Jr, Kyriakides T, Johnson A, Garcia E, Buhimschi CS, Buhimschi IA: Histologic changes of the 
fetal membranes after fetoscopic laser surgery for twin-twin transfusion syndrome. Pediatr Res 2015, 78:247-255

25. Menon R, Richardson LS: Preterm prelabor rupture of the membranes: a disease of the fetal membranes. Semin Perinatol 2017, 41: 409-419

26. Janzen C, Sen S, Lei MY, Gagliardi de Assumpcao M, Challis J, Chaudhuri G: The role of epithelial to mesenchymal transition in human amniotic membrane rupture. J Clin Endocrinol Metab 2016, 102:1261-1269

27. Mogami H, Hari Kishore A, Akgul Y, Word RA: Healing of preterm ruptured fetal membranes. Sci Rep 2017, 7:13139

28. Sheller S, Papaconstantinou J, Urrabaz-Garza R, Richardson L, Saade G, Salomon C, Menon R: Amnion-epithelial-cell-derived exosomes demonstrate physiologic state of cell under oxidative stress. PLoS One 2016, 11:e0157614

29. Moreno-Bueno G, Peinado H, Molina P, Olmeda D, Cubillo E, Santos V, Palacios J, Portillo F, Cano A: The morphological and molecular features of the epithelial-to-mesenchymal transition. Nat Protoc 2009, 4:1591-1613

30. Itahana K, Campisi J, Dimri GP: Methods to detect biomarkers of cellular senescence: the senescence-associated beta-galactosidase assay. Methods Mol Biol 2007, 371:21-31

31. Behnia F, Taylor BD, Woodson M, Kacerovsky M, Hawkins H, Fortunato SJ, Saade GR, Menon R: Chorioamniotic membrane senescence: a signal for parturition? Am J Obstet Gynecol 2015, 213: 359.e1-16

32. Dixon CL, Richardson L, Sheller-Miller S, Saade G, Menon R: A distinct mechanism of senescence activation in amnion epithelial cells by infection, inflammation, and oxidative stress. Am J Reprod Immunol 2018, 79

33. Fernando RI, Castillo MD, Litzinger M, Hamilton DH, Palena C: IL-8 signaling plays a critical role in the epithelial-mesenchymal transition of human carcinoma cells. Cancer Res 2011, 71: $5296-5306$

34. Rennekampff HO, Hansbrough JF, Kiessig V, Dore C, Sticherling M, Schroder JM: Bioactive interleukin- 8 is expressed in wounds and enhances wound healing. J Surg Res 2000, 93:41-54

35. Kim MS, Lee MH, Kwon BJ, Kim D, Koo MA, Seon GM, Park JC: Homogeneity evaluation of mesenchymal stem cells based on electrotaxis analysis. Sci Rep 2017, 7:9582

36. Lappas M, Hiden U, Desoye G, Froehlich J, Hauguel-de Mouzon S, Jawerbaum A: The role of oxidative stress in the pathophysiology of gestational diabetes mellitus. Antioxid Redox Signal 2011, 15: 3061-3100

37. Asea A, Jean-Pierre C, Kaur P, Rao P, Linhares IM, Skupski D, Witkin SS: Heat shock protein-containing exosomes in mid-trimester amniotic fluids. J Reprod Immunol 2008, 79:12-17

38. Pierce J: Collection and characterization of amniotic fluid from scheduled C-section deliveries. Cell and Tissue Banking 2016, 17 : 413-425

39. Behnia F, Peltier MR, Saade GR, Menon R: Environmental pollutant polybrominated diphenyl ether, a flame retardant, induces primary amnion cell senescence. Am J Reprod Immunol 2015, 74: 398-406
40. Landen NX, Li D, Stahle M: Transition from inflammation to proliferation: a critical step during wound healing. Cell Mol Life Sci 2016, 73:3861-3885

41. Litwiniuk M, Grzela T: Amniotic membrane: new concepts for an old dressing. Wound Repair Regen 2014, 22:451-456

42. Zhao B, Liu JQ, Zheng Z, Zhang J, Wang SY, Han SC, Zhou Q, Guan H, Li C, Su LL, Hu DH: Human amniotic epithelial stem cells promote wound healing by facilitating migration and proliferation of keratinocytes via ERK, JNK and AKT signaling pathways. Cell Tissue Res 2016, 365:85-99

43. Moore RM, Mansour JM, Redline RW, Mercer BM, Moore JJ: The physiology of fetal membrane rupture: insight gained from the determination of physical properties. Placenta 2006, 27:1037-1051

44. Menon R, Fortunato SJ, Milne GL, Brou L, Carnevale C, Sanchez SC, Hubbard L, Lappas M, Drobek CO, Taylor RN: Amniotic fluid eicosanoids in preterm and term births: effects of risk factors for spontaneous preterm labor. Obstet Gynecol 2011, 118:121-134

45. Maymo JL, Riedel R, Perez-Perez A, Magatti M, Maskin B, Duenas JL, Parolini O, Sanchez-Margalet V, Varone CL: Proliferation and survival of human amniotic epithelial cells during their hepatic differentiation. PLoS One 2018, 13:e0191489

46. Lee JM, Dedhar S, Kalluri R, Thompson EW: The epithelialmesenchymal transition: new insights in signaling, development, and disease. J Cell Biol 2006, 172:973-981

47. Chaffer CL, Brennan JP, Slavin JL, Blick T, Thompson EW, Williams ED: Mesenchymal-to-epithelial transition facilitates bladder cancer metastasis: role of fibroblast growth factor receptor-2. Cancer Res 2006, 66:11271-11278

48. Laurila P, Virtanen I, Lehto VP, Vartio T, Stenman S: Expression and distribution of vimentin and keratin filaments in heterokaryons of human fibroblasts and amnion epithelial cells. J Cell Biol 1982, 94: 308-315

49. Regauer S, Franke WW, Virtanen I: Intermediate filament cytoskeleton of amnion epithelium and cultured amnion epithelial cells: expression of epidermal cytokeratins in cells of a simple epithelium. J Cell Biol 1985, 100:997-1009

50. Richardson L, Martin L, Menon R: Characteristics, properties, and functionality of fetal membranes: an overlooked area in the field of parturition. Encyclopedia of Reproduction, ed 2. Amsterdam, the Netherlands: Elsevier, 2018. pp. 387-398

51. Faulk WP, Matthews R, Stevens PJ, Bennett JP, Burgos H, Hsi BL: Human amnion as an adjunct in wound healing. Lancet 1980, 1: $1156-1158$

52. Liu CY, Lin HH, Tang MJ, Wang YK: Vimentin contributes to epithelial-mesenchymal transition cancer cell mechanics by mediating cytoskeletal organization and focal adhesion maturation. Oncotarget 2015, 6:15966-15983

53. Mendez MG, Kojima S, Goldman RD: Vimentin induces changes in cell shape, motility, and adhesion during the epithelial to mesenchymal transition. Faseb J 2010, 24:1838-1851

54. Nery FC, Zeng J, Niland BP, Hewett J, Farley J, Irimia D, Li Y, Wiche G, Sonnenberg A, Breakefield XO: TorsinA binds the KASH domain of nesprins and participates in linkage between nuclear envelope and cytoskeleton. J Cell Sci 2008, 121:3476-3486 